\title{
Privacy and Auditability in Cloud Assisted Health Data
}

\author{
K. Vijaya Swetha, P. Sai Kiran and K.V.V. Satyanarayana \\ Department of Computer Science and Engineering, Koneru Lakshmaiah, \\ University, Vaddeswaram, Andhra Pradesh, India
}

\begin{abstract}
Cloud security is an emerging concept in data outsourcing in the cloud and also maintaining of multiple copies of each user in different servers is crucial task in data outsourcing in terms of customer cost, memory storage in multi user access in real time distributed systems. Traditionally a Map-Based Provable Multicopy Dynamic Data Possession (MB-PMDDP) plan that has the accompanying components: it gives a proof to the customers can utilize that the CSP is not unfaithful by sparing less duplicates, it encourages outsourcing of intense data, i.e., it encourages piece level capacities, for example, forestall modification, arrangement, evacuation and append and it permits endorsed customers to effortlessly get to the information record copies spared by the CSP. For example consider e-Health care systems for providing security to patients sensitive information with modifications (like insert, deletion, updation) by different peoples and store those files in different servers causes loss of security to patients because of corrupted data in different servers. To support above considerations in multi user data access in this study, we propose to develop, incorporate solace with versatile therapeutic consideration frameworks with the assistance of the private evaluation. Our framework offers critical components, for example, successful key control, security, safeguarding data storage room and recuperation, particularly for recuperation at crisis circumstances and auditability for abusing wellbeing data. Especially, we prescribe to consolidate key control from the pseudo interesting number maker for unlinkability, a sheltered and secured posting technique for solace ensuring catchphrase and key expression search for which disguises both search for and access designs taking into account repetition.
\end{abstract}

Key words: Access control, e-Health, cloud service provider, data redundancy, dynamic environment, modifications

\section{INTRODUCTION}

Out looking for subtle elements to an internet Cloud Service Provider (CSP) permits organizations to store a larger number of points of interest on the CSP than on individual PCs. Such outsourcing of points of interest storage room empowers organizations to focus on improvements and diminishes the weight of consistent server up-dates and other preparing problems. Once the subtle element has been contracted to an online CSP which may not be dependable, the points of interest proprietors lose the direct administration over their fragile points of interest. This absence of administration raises new impressive and confounded ventures identified with subtle elements security and dependability assurance in preparing. The security issue can be taken care of by encoding fragile points of interest before outsourcing to inaccessible web servers. All things considered, it is an urgent interest of clients to have a solid proof that the web servers still have their points of interest and it is not being meddled with or somewhat uprooted after some time. Thusly, numerous analysts have concentrated on the issue of Provable Data Possession (PDP) (Barsoum and Hasan, 2015) and proposed distinctive plans to survey the subtle elements put away on inaccessible web servers. A guide

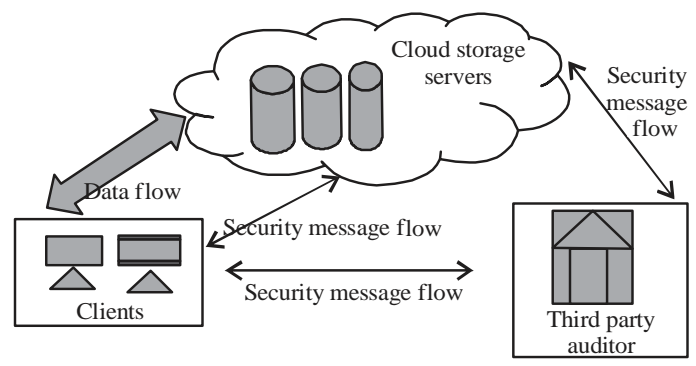

Fig. 1: Cloud data storage system with outsourced data

based provable a Map-Based Provable Multi-copy Dynamic Data Possession (MB-PMDDP) plan. Constructed gives a sufficient assurance that the CSP shops all copies that are settled upon in the bolster contract as shown in Fig. 1.

In addition, the arrangement encourages outsourcing of intense points of interest, i.e., it encourages piece level capacities, for example, forestall adjustment, position, evacuation and add. The approved clients, who have the privilege to availability the proprietor's record can without much of a stretch openness the copy got from the CSP. 
Asian J. Inform. Technol., 18 (4): 133-138, 2019

There are clarifications for keeping medicinal subtle elements individual and confining the availability. An organization might choose not to contract somebody with specific sicknesses. The security arrangement, organization might decline to give protection approach, scope knowing the malady history of a patient. In spite of the fundamental significance, solace issues are not tended to suffice at the specialized stage and endeavors to keep wellbeing points of interest secure have frequently missed the mark. This is on the grounds that securing solace in the internet is fundamentally more muddled. Subsequently, there is a critical requirement for the improvement of suitable strategies, models and methods guaranteeing protection and security to defend sensitive and individual computerized data. Outsourcing points of interest storage room and computational activities gets to be a developing pattern based on design pattern present cloud. A significantly frightful tale is that the organization's Total Claims Control and Capture (TC3) which offers claim administration awesome alternatives for medicare payers, as an example, medicare payers, safety technique offices, urban regions and self-guaranteed employer fitness arranges. TC3 has been utilizing Amazon's EC2 wondering to system the diffused factors their customers send in (a huge wide variety of proclamations daily) which include fragile health points of the hobby. Outsourcing the computations to the spares TC3 from purchasing and keeping up web servers and permits TC3 to exploit Amazon's aptitude to prepare and assess subtle elements speedier and all the more productive.

The proposed cloud-helped the cell wellbeing systems administration is motivated by the force, adaptability, comfort and cost proficiency of the cloud-based information/calculation outsourcing model. We present the individual assurance which can be considered as a bolster offered to sell clients. The proposed choices are based on the bolster plan appeared in Fig. 2. A product as a bolster (SaaS) organization gives individual assurance administrations by utilizing the offices of the group suppliers (e.g., Amazon, Google). Versatile clients delegate PC tasks to the private Evaluation which shops the handled, resulting in a general society cloud. The cloud-helped bolster a plan props up execution of pragmatic solace frameworks since escalated computations and storage room can be moved to the customers individual evaluation, leaving cell clients with lightweight tasks.

Literature review: Some early takes a shot at the solace insurance for e-wellbeing data concentrate on the structure outline (Tong et al., 2014; Deswarte et al., 2004; Sebe et al., 2008; Golle et al., 2002), for example, the sort of advantage of solace for e-wellbeing procedures, the check contingent upon current Wi-Fi offices, the part based strategy for openness restrictions and so forth. Particular, Individual Based Encryption (IBE)

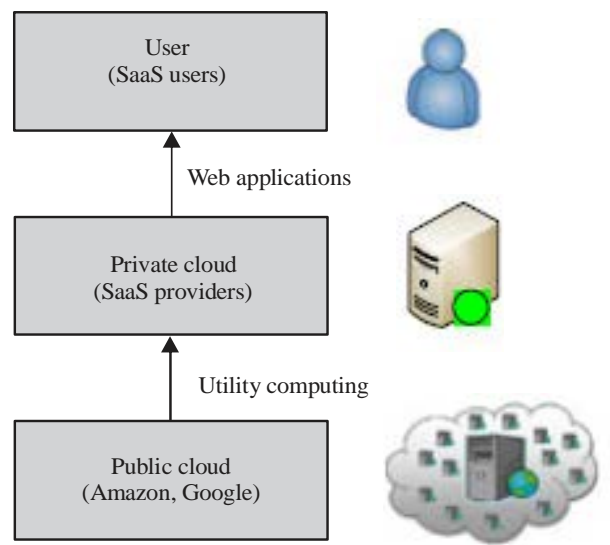

Fig. 2: SaaS Model with cloud security in private and public cloud

(Shah et al., 2007) has been utilized (Deswarte et al., 2004) for executing simple element based cryptographic openness control. A number of the predominant activities on e-wellness solace, Healthcare Information Privacy Assurance (HIPA) tested out the critics and unique demanding situations of medicinal information solace and the overpowering safety infringement records that lead from poor assisting mechanical improvement. HIPA was one of the initial few assignments that wanted to make solace mechanical advancement and security ensuring bases to fulfill upgrading any antagonistic health data framework in which people can secure their own data. We took over our line of examination (Wang et al., 2010) with different partners and portrayed the assurance particulars for e-wellbeing procedures by Ateniese et al. (2008).

\section{MATERIALS AND METHODS}

Searchable symmetrical encryption: SSE permits data proprietors to store secured records on far off server which is made as fair yet inquisitive festival and in the meantime gives a way to look over the secured records. All the extra basically, neither the potential of outsourcing nor catchphrase and key expression searching could result in any statistics move to any festival other than the statistics proprietor in the end completing a legitimate assurance of solace. SSE changed into first advanced by way of Filho and later upgraded through (Sebe et al., 2008), at a propelled level, SSE includes the accompanying techniques.

Keygen (s): This image is utilized by clients to create insider statistics of instate the arrangement. It calls for the insurance parameters and consequences a key okay.

BuildIdx (D, k): The customer works this picture to manufacture the files, indicated by using I, for a will 
power of papers D. It calls for the important thing ok and $\mathrm{D}$ and consequences I, through which papers can be retrievable at the same time as staying secure.

Trapdoor (K, w): The purchaser works this work to assess a trapdoor for a catch phrase and key expression w, permitting searching for this watchword and key expression. A trapdoor Tw can likewise be considered as an intermediaries for $\mathrm{w}$ all together to conceal the genuine essentialness of $\mathrm{w}$. Thusly, Tw ought to stream the insights about $\mathrm{w}$ as meager as could be expected under the circumstances. The work takes the key $\mathrm{K}$ and the watchword and key expression $\mathrm{w}$ and results the individual trapdoor Tw.

Search (I, Tw): This research is executed by the removed server to discover records containing the client depicted watchword and key expression w. Because of the utilization of the trapdoor, the server can do the particular inquiry without comprehension the real watchword and key expression. The images require the fabricated secured list I and the trapdoor Tw and effects the identifiers of facts records which contains catchphrase and key expression w. Solidly in Curtmola et al., s. development, each study is brought by an identifier and matches a hub. All statistics in D are secured and held in the inaccessible web servers.

\section{MB-PMDDP scheme for security}

Synopsis and rationale: Producing elite differentiable copies of the computer records file is the middle to add to a provable multi-replica facts proprietorship association. Indistinguishable copies let the CSP to essentially misdirect the owner by means of sparing one and most effective reproduction and appearing that it stores numerous duplicates. Using an easy yet a hit manner, the proposed plant produces elite copies, making use of the dissemination living association of any comfortable protection arrangement. The dissemination domestic ensures that the result bits of the cipher text rely upon the grievance bits of the plaintext in an exceedingly confounded manner, i.e., there might be an erratic finishing touch rotation of the cipher text, if there is a single piece shift in the plaintext (Erway et al., 2009). The institutions between the recommended customers and the CSP is respected through this gadget of creating restrictive copies wherein the preceding can unscramble/get right of entry to facts copy got from the CSP. In the proposed arrangement, the affirmed clients require simply to keep an single key (imparted to the record owner) to unscramble the file copy and it isn't by any stretch of the creativeness to differentiate the listing of them were given replica.

Within the challenge, we propose a MB-PMDDP association allowing the facts proprietor to overtake and variety the avoids of facts document duplicates outsourced to questioning internet servers which are probably untrusted. Accepting such copies of effective facts wishes the comprehension of the piece releases to ensure that the facts anticipates in all copies are dependable with the more recent types from the owner. Similarly, the verifier has to consider the square lists to certify that the CSP has put or included the new anticipates on the asked parts in all copies.

\section{MB-PMDDP step-by-step steps}

Key generation: Let ^e: G1×G2 $\rightarrow \mathrm{GT}$ be a bilinear guide and $g$ a maker of G2. The data proprietor works the Key Gen criteria to create an individual key $\mathrm{x} \in \mathrm{Zp}$ and a group key $\mathrm{y}=\mathrm{gx} \in \mathrm{G} 2$.

Creation of distinct copies: For data $F=\{b j\} 1 \leq j \leq m$, the proprietor works the CopyGen criteria to make $n$ differentiable duplicates $\mathrm{F}=\{\mathrm{Fi}\} 1 \leq \mathrm{i} \leq \mathrm{n}$ where a duplicate $\mathrm{Fi}=\left\{{ }^{\sim} \mathrm{bi} j\right\} 1 \leq \mathrm{j} \leq \mathrm{m}$. The avert ${ }^{\mathrm{bi}} \mathrm{j}$ is created by connecting a copy assortment $\mathrm{i}$ with the avert $\mathrm{b} \mathrm{j}$, then scrambling utilizing a security arrangement EK, i.e., ` bi $\mathrm{j}=\mathrm{EK}$ (i\|b j). The secured avert $\mathrm{bi} \mathrm{j}$ is divided into s parts $\left\{{ }^{\sim} \mathrm{bi}\right.$

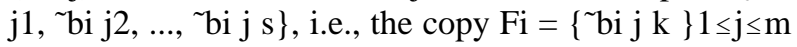
$1 \leq \mathrm{k} \leq \mathrm{s}$ where every industry bi $\mathrm{j} \mathrm{k} \in \mathrm{Zp}$ for some colossal essential p.

The assortment of averting regions s depends upon on the anticipate size furthermore, the essential p where $\mathrm{s}=\mid$ block size $/ /|\mathrm{p}|$ (|.| is the bit length). The endorsed clients require just to keep an individual mystery key K. Later, when a qualified client gets data duplicated from the CSP, he decodes the copy forestalls, dispenses with the duplicate index from the forestall features and after that recombines the decoded forestalls to revamp the fundamentally.

MB-PMDDP arrangement encourages group unquestionable status where anybody who knows the proprietor's group key yet is not so much the data proprietor can convey an issue vector to the CSP and affirm the response. Open evidence can alter clashes that might happen between the data proprietor and the CSP with respect to data unwavering quality. In the event that such a contention happens, a Third Party Auditor (TPA) can make sense of whether the data unwavering quality are overseen or not. Subsequent to the proprietor's group key is just expected to complete affirmation step, the real is not required to demonstrate his mystery key to the TPA.

Cloud-assisted privacy-preserving e-Health: Our cloud-helped security saving portable therapeutic consideration framework contains two parts: retrievable assurance and auditable accessibility control. After getting the data from customers, the individual speculation strategies and shops it on gathering details such that capacity region space accommodation and viable 
Asian J. Inform. Technol., 18 (4): 133-138, 2019

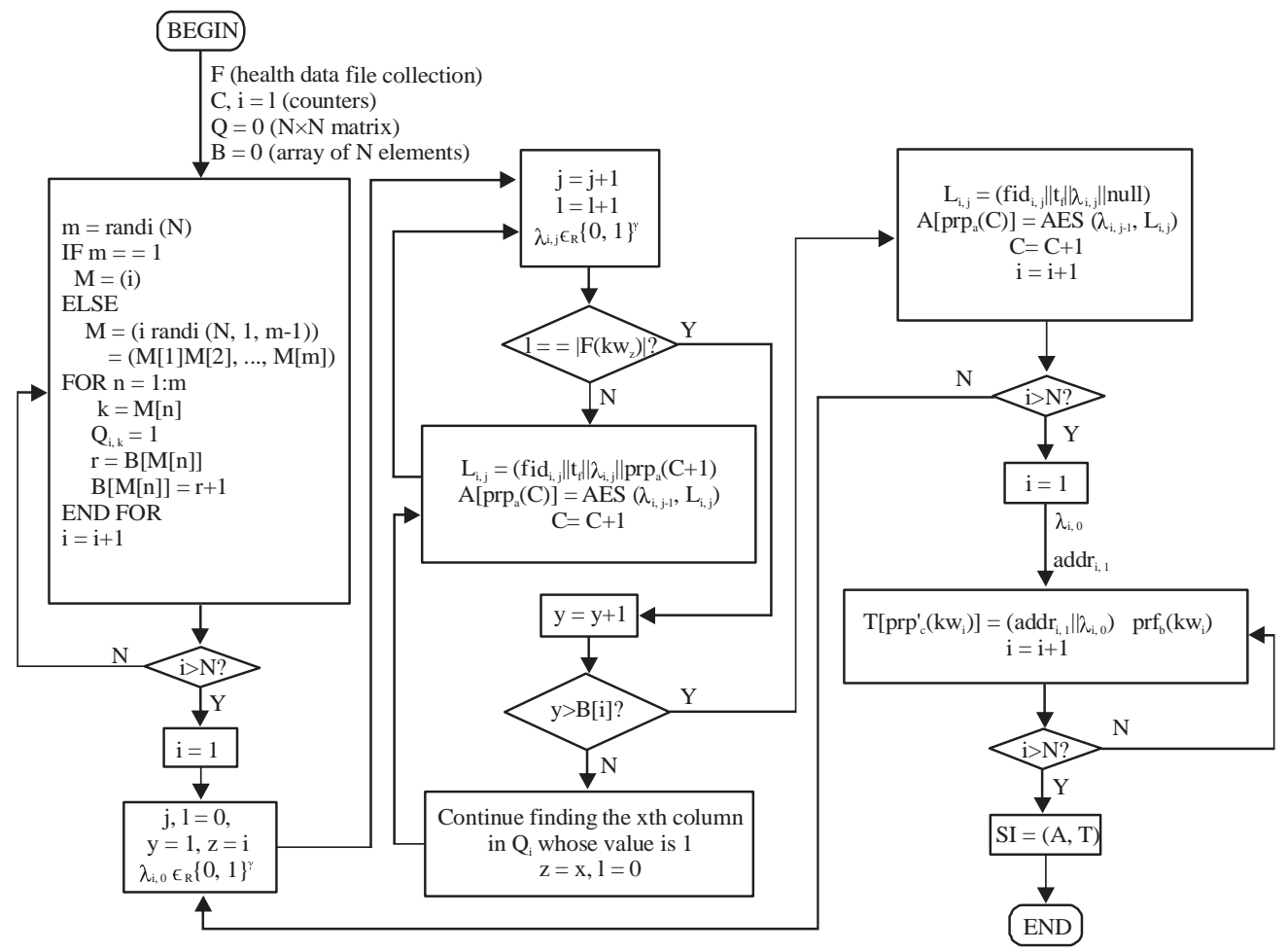

Fig. 3: Pattern hiding secure index

reclamation can be certain. Next, the individual speculation participates in the bootstrapping of data accessibility and auditability arrangement with customers, so, it can later follow up on the client's part to practice accessibility control and survey on acknowledged occasions.

Capacity comfort and efficient retrieval: The primary component is capacity zone space comfort for the data. Our capacity ranges, space procedure is relying upon securing accumulation or SSE, so that, the customer can secure the data with more data segments to take into account powerful search for. It has been demonstrated that the safe list based system is appealing among various methodologies for capacity zone space comfort. In sorts, the individual deduction takes the piece of the customer, and town deduction is the capacity territory space server in SSE.

In any case, there are sensible conditions were unverifiable (Wang et al., 2009; Barsoum and Hasan, 2015) which we will manage in this record. The unlinkability need was not very much settled. Nothing, unless there are other option works portrayed how to make the record identifiers. In the event that the identifiers keep certain style, it will be simple for the assailants to construe that few data are from a same customer (Fig. 3).

Manifestly, we require identifiers that display up irregular yet may be effortlessly taken care of. In recurring SSE, all placed away facts are correctly secured using the equal key. This isn't a legitimate insurance fashion subsequent to the greater we utilize a key, the more records the aggressors can get to interrupt the key. We alongside these traces want to improve the important thing as often as sufficiently feasible to keep a strategic distance from the important thing wear-out. To accomplish quick and successful rebuilding, it is right to build the information such that they can be regarded via. the date/time of development, aside from the watchwords and phrases. That is especially, helpful in crucial situations in which looking may be restricted down to the maximum information. Searching based on a date/time has to be looked after otherwise from catchphrases seeing that date/time isn't definitely touchy statistics and the security need may be casual for productivity. Not one of the current fitting works (Hao et al., 2011; Barsoum and Hasan, 2015; Hao and Yu, 2010) may want to stow away the look for or accessibility fashion as in advance portrayed. The primary SSE tactics that hide each outlines are prescribed through Goldreich what is greater, (Mykletun et al., 2006). Those outlines are deliberating now not conscious RAMs and are to an incredible diploma vain due the round many-sided best.

In our style, customers don't at ease, their well being statistics making use of ABE. The statistics are secured utilizing the amazingly capable approach depicted in our space for capability solace aspect. As an alternative, customers use $\mathrm{ABE}$ to secure the important thing shares, 
Asian J. Inform. Technol., 18 (4): 133-138, 2019

so that, simply advocated activities can decode them and produce actual marks. The person questioning and EMT will edge sign the data availability request displayed by means of the EMT which incorporates the quest queries and time move the EMT needs to searching for. The customer can check the hobby and the validity of the brink trademark to survey the accompanying at a later time: the interest turned into due to a true therapeutic dire, the EMT has asked facts just pertinent to the remedy, the EMT cannot decline the facts hobby and openness if either or is damaged and the person questioning cannot inaccurately price the EMT if neither nor is ruptured. In doing as such clients avoid the trying out method of distinguishing who can openness which records report (s). Alternatively, they just need to make feel of who can availability their information and allot a key examine correspondingly. Whether an affirmed birthday party has legitimately used the facts is left to the auditability in our style. We likewise offer make usage of the cutting-edge medical offerings framework shape to confirm the validity of the element.

\section{RESULTS AND DISCUSSION}

Performacne evaluation: Here, we examine the productiveness of the exhibited plans: MB-PMDDP and TB-PMDDP. The data document $F$ utilized as part of our productivity exploration is of measurement $64 \mathrm{MB}$ with $4 \mathrm{~KB}$ anticipate measurement. Without lack of sweeping declaration, we take delivery of that the coveted safety stage is 128-piece. In the end, we use an elliptic curve portrayed over Galois field pieces (a factor on this twist can be regarded by way of 257 portions utilizing compacted mirrored image) and a cryptographic hash of measurement 256 pieces (e.g., SHA-256). Like (Hao and $\mathrm{Yu}, 2010$ ) the count value is classed regarding the utilized crypto-operations that are documented in Table 1. G indicates a set of focuses over an appropriate elliptic twist inside the bilinear coupling.

Allow $n, m$ and s suggest the quantity of copies, the amount of anticipating in step with copy and the amount of territories in keeping with averted, one at a time. permit c method the amount of anticipates to be driven and size of the facts document copy. Let the imperative additives utilized with the PRP and the PRF be of measurement 128 portions. Work area I shows a hypothetical examination for the establishment, storage room, correspondence, estimations and effective capacities expenses of the two plans: MB-PMDDP and TB-PMDDP. We assess the storage room and communication effectiveness by taking a gander at the storage room and association running expenses amid data outsourcing and recuperation. The cost is portrayed to be any data that gives the motivation behind administration, assurance, bookkeeping and so forth, yet the vital medicinal consideration data or its security in terms of time efficiency as following in Table 1.
Table 1: Comparison results to ABE and MB-PMDDP with respect to time in file storage

\begin{tabular}{lcl}
\hline File Id's & MB-PMDDP & ABE \\
\hline 1 & 4.3 & 3.7 \\
2 & 3.8 & 2.9 \\
3 & 3.2 & 2.7 \\
4 & 2.9 & 2.4 \\
5 & 1.8 & 1.2 \\
6 & 1.2 & 0.68 \\
\hline
\end{tabular}

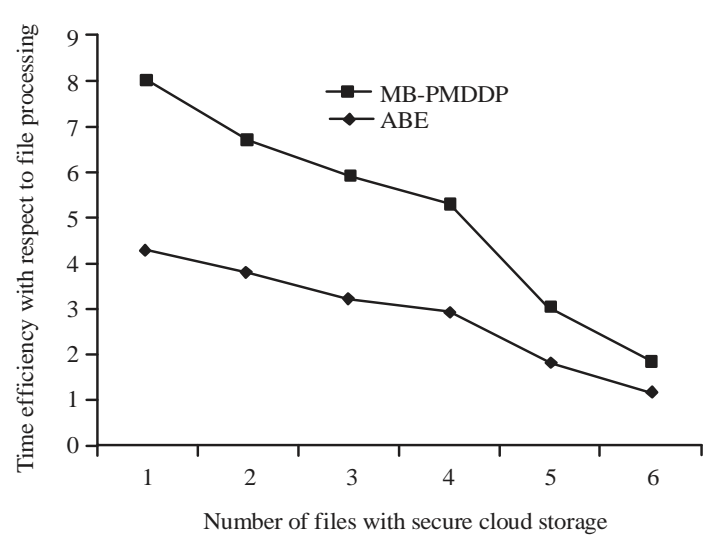

Fig. 4: Comparisons analysis of two proposed techniques with respect to time

We likewise explore the collaboration cost amid an EMT's data request with a fruitful recuperation. For quality, we separate the collaboration into two sections, i.e., cooperation between data requesters, for example, EMT and the individual assurance and that between the individual assurance and people in general reasoning. From the examination above, we know that the storage room cost is a straight line with the amount of contract restorative consideration data, while the communication cost can be considered as nonstop per data request. The outcome demonstrates that the recommended arrangement is viable and additionally versatile.

In this research, we check the computational skill ability of the proposed techniques. In particular, we are keen on whether our techniques are a hit whilst cellular telephones are locked in, i.e., sufferers setting up the security, safeguarding garbage room and EMTs getting the medicinal consideration statistics in disaster instances. We related our strategies making use of Samsung Nexus $S$ cell phones (1-GHz Cortex-A8, 512-MB RAM) and computed the playback. For executions of IBE and ABE, we utilized the espresso paring-based cryptography series and applied a matching nicely disposed type A one hundred sixty-piece elliptic twist organization.

In security protecting storage room using individual mobile phones, viable mystery key capacities are for the most part connected with which we won't concentrate on in the evaluation as shown in above Fig. 4. In crisis restorative consideration data openness using EMT mobile phones, the most excessive continuous counts 
incorporates IBE decoding and ABE unscrambling, producing a successive trademark on components and a constrained point of confinement trademark on the availability request and affirming as far as possible trademark from the individual assurance. Be that as it may, IBE unscrambling, ABE decoding and successive trademark can be performed for the last time openness for the same person which is helpful if the EMT will issue numerous availability requests. Regardless, we consider this cost following an EMT is prone to openness a patient's therapeutic consideration data just once as a rule.

\section{CONCLUSION}

Data outsourcing in cloud computing is emerging concept in real time data sharing between users in e-Health care systems for processing efficient privacy in data storage. In this study different authentication owners perform and store the same data with multiple copies in single server management for accessing multi files in configuration of different user's performance. Then security is the main factor for storage of multiple files in single server for processing several data events in real time distributed computing. In this study, we propose $\mathrm{ABE}$ for processing security to multi-copy storage in real time communication systems. Our experimental results show efficient security in reliable data storage.

\section{REFERENCES}

Ateniese, G., R. di Pietro, L.V. Mancini and G. Tsudik, 2008. Scalable and efficient provable data possession. Proceedings of the 4th International Conference on Security and Privacy in Communication Networks, September 22-25, 2008, New York, USA., pp: 1-11.

Barsoum, A.F. and M.A. Hasan, 2015. Provable multicopy dynamic data possession in cloud computing systems. IEEE. Trans. Inf. Forensics Secur., 10: 485-497.

Deswarte, Y., J.J. Quisquater and A. Saidane, 2004. Remote Integrity Checking. In: Integrity and Internal Control in Information Systems VI. Jajodia, S. and L. Strous (Eds.). Springer US, Berlin, Germany, ISBN: 978-1-4020-7900-9, pp: 1-11.

Erway, C., A. Kupcu, C. Papamanthou and R. Tamassia, 2009. Dynamic provable data possession. Proceedings of the 16th ACM Conference on Computer and Communications Security, November 9-13, 2009, Chicago, IL., USA., pp: 213-222.
Golle, P., S. Jarecki and I. Mironov, 2002. Cryptographic Primitives Enforcing Communication and Storage Complexity. In: Financial Cryptography. Matt, B. (Ed.). Springer Berlin Heidelberg, Berlin, Germany, ISBN: 978-3-540-00646-6, pp: 120-135.

Hao, Z. and N. Yu, 2010. A multiple-replica remote data possession checking protocol with public verifiability. Proceedings of the 2010 Second International Symposium on Data, Privacy and E-Commerce (ISDPE), September 13-14, 2010, IEEE, Buffalo, New York, USA., ISBN: 978-1-4244-8377-8, pp: 84-89.

Hao, Z., S. Zhong and N. Yu, 2011. A privacy-preserving remote data integrity checking protocol with data dynamics and public verifiability. IEEE Trans. Knowl. Data Eng., 23: 1432-1437.

Mykletun, E., M. Narasimha and G. Tsudik, 2006. Authentication and integrity in outsourced databases. ACM. Trans. Storage TOS., 2: 107-138.

Sebe, F., F.J. Domingo, B.A. Martinez, Y. Deswarte and J.J. Quisquater, 2008. Efficient remote data possession checking in critical information infrastructures. IEEE. Trans. Knowl. Data Eng., 20: 1034-1038.

Shah, M.A., M. Baker, J.C. Mogul and R. Swaminathan, 2007. Auditing to keep online storage services honest. Proceedings of the 11th USENIX Workshop Hot Topics Operational System HOTOS, May 7-9, 2007, HOTOS, Catamaran Resort Hotel, San Diego, California, pp: 1-6.

Tong, Y., J. Sun, S.S. Chow and P. Li, 2014. Cloud-assisted mobile-access of health data with privacy and auditability. IEEE. J. Biomed. Health Inf., 18: 419-429.

Wang, C., Q. Wang, K. Ren and W. Lou, 2010. Privacy-preserving public auditing for data storage security in cloud computing. Proceedings of the 2010 IEEE Conference on INFOCOM, March 14-19, 2010, IEEE, San Diego, California, pp: 1-9.

Wang, Q., C. Wang, J. Li, K. Ren and W. Lou, 2009. Enabling Public Verifiability and Data Dynamics for Storage Security in Cloud Computing. In: Computer Security. Backes, M. and P. Ning (Eds.). Springer Berlin Heidelberg, Berlin, Germany, ISBN: 978-3-642-04443-4, pp: 355-370. 